\title{
Advances in Network-based Information Systems
}

\author{
Ilsun You ${ }^{\mathrm{a}}$, Kangbin Yim ${ }^{\mathrm{b}}$ and Leonard Barolli ${ }^{\mathrm{c}, *}$ \\ ${ }^{a}$ School of Information Science, Korean Bible University, Seoul, Korea \\ ${ }^{\mathrm{b}}$ Department of Information Security Engineering, Soonchunhyang University, Asan, Korea \\ ${ }^{\mathrm{c}}$ Department of Information and Communication Engineering, Fukuoka Institute of Technology, \\ Fukuoka, Japan
}

Tremendous advances in the information systems and the communication technologies for the last two decades have made our society an all-connected network environment. Stand-alone or stationarynetworked information systems from the first decade have been integrated into small chips or devices as system-on-chips. In particular, the wireless communication network has superimposed on the existing wired network over the various mobile devices during the last decade. Additionally, different types of access networks have been deployed and almost every object is becoming connected to the single global network throughout the world. As a result, the information systems connected to the single network now may span from RFID barcodes, biomedical sensors, personal or home entertainment devices and automated office machines to environmental surveillance systems, disaster management systems, military equipment, and space satellites.

In regards to the network as the infrastructure of the all-connected environment, several essential aspects are required to be considered: addressing, routing, quality control, admission control, unified interface and security. Similarly, issues on unique identification, end-to-end connectivity, protocol adaptation, and homogeneous access also need to be thoroughly discussed. This special issue looked for several high quality research results with outstanding theoretical and practical contributions to the technological advancements of the network-based information systems within the various unfolded technological aspects listed above.

To meet the goal, the papers in this special issue were distilled from the papers presented in the 14th International Conference on Network-based Information Systems (NBiS-2011), held in Tirana, Albania. Since this event started in 1998, it has been throwing a large effort to bring together researchers from both network and information systems with the aim of encouraging the exchange of ideas and experiences between these two communities. The selected six outstanding papers have been expanded and revised through a strict review process for months.

\footnotetext{
${ }^{*}$ Corresponding author: Leonard Barolli, Department of Information and Communication Engineering, Fukuoka Institute of Technology, Fukuoka, Japan. E-mail: barolli@fit.ac.jp.
} 
The first paper [1] "Glowbal IP: an adaptive and transparent IPv6 integration in the Internet of Things" by Antonio J. Jara, Miguel A. Zamora and Antonio F. G. Skarmeta proposes a technology to adapt the existing legacy protocols to IPv6. There is no doubt that the IPv6 framework is an essential infrastructure for the all-connected global network to support addressing the individual devices around the world and delivering controls and data among them, especially to realize the Internet of Things. However, different types, dimensions and features of the existing devices, connections, protocols, and services have been huddling up and down or side-by-side and they make it difficult to consistently and intuitively access each other among the heterogeneous entities. To resolve this complex situation, this paper designs an efficient scalable protocol conversion specification that offers global interoperability without too much processing overhead. The empirical result from the implementation of an adaptation between 6LoWPAN and IPv6 shows that the Web-based common service over the implementation can provide a reasonable performance.

In a dynamic network integrated with mobile devices, the efficiency of the routing protocol is a significant factor for the performance of the network in the sense of communication overhead, link throughput, and end-to-end delay. The second paper [2] "Condensation-Based Routing in Mobile AdHoc Networks" from Francesco Palmieri and Aniello Castiglione introduces an adaptive probabilistic routing technique for broadcast-based reactive routing protocols in MANETs. Against the proactive routing protocols, which consistently update the routing information and ensure a guaranteed end-to-end delay at the cost of larger control traffic, the reactive routing protocols generate a less traffic overhead by initiating a route discovery process in an on-demand fashion, even though it causes a significant latency before setting up a route. To resolve this problem, the proposed protocol utilizes the probabilistic features of the link status within moving nodes such as signal power, noise level, and total number of links in broadcasting the routing control messages. According to the performance evaluation using an event simulator, the protocol provides higher throughput and lower end-to-end delay, especially in a denser network.

Networks need to have efficient traffic management policies to support the quality control for the multimedia services among the networked entities. In particular, the network that imposes packet loss should have a technology to compensate the loss for a robust quality media transmission. The third paper [3] from Xingjun Zhang, Cuiping Jing, Feilong Tang, Scott Fowler, Huali Cui, and Xiaoshe Dong, entitled "Joint Redundant and Random Network Coding for Robust Video Transmission over Lossy Networks" proposes a novel network-coding scheme that combines the redundant and the random network coding algorithms. The redundant network coding encodes the media packets to enhance the robustness of the lossy network, which reduces the error rate. The random network coding encodes the packets to enhance the network bandwidth utilization, which reduces the end-to-end delay and then compensates the traffic redundancy. They also proved the feasibility of the proposed scheme by implementing a prototype and deriving a reasonable result from an empirical study.

As the information systems become versatile and portable, multi-modal interface is becoming another common characteristics in the Human-Computer Interface area. The intuitiveness also needs to be achieved on the user interface in this multi-device and multi-modal environment. In particular, autonomous selection of services requires a scalable cognitive algorithm for context or ambience awareness based on the user interactions. The next paper [4] "Context-aware HCI Service Selection" by Yao Shen, Minjie Wang, Xiaoxin Tang, Yi Luo, and MinyiGuo proposes a service-oriented framework for interaction migration and context-driven service selection in the multi-modal environment. The intelligent service-matching algorithm consists of three different processes for service properties, user preferences, 
and environmental contexts within devices and locally calculates the matching degree for service selection. The context manager performs a global service selection based on the matching degrees from multiple devices to combine a best set of services for users.

Anonymous authentication and signature between entities are very important in a large scaled mobile network environment, especially when the attributes of the member entities are diversiform and individual privacies are concentrated. In the fifth paper [5] "Flexible Multi-Authority Attribute-based Signature Schemes for Expressive Policy," Dan Cao, BaokangZhao, Xiaofeng Wang and Jinshu Su propose two multi-authority attribute-based signature schemes, TR_MABS and DNF_MABS. The attribute-based signature scheme allows users to sign a message with their own attributes that are obtained from the trusted authority. The existing attribute-based signature schemes use a single attribute authority and the privacy problem may arise through leakage of the attributes. Instead, the proposed schemes use multiple distinct authorities to satisfy the requirement of the real world applications. The proposed TR_MABS scheme, which introduces a flexible way to express the policy of the authority by adapting an attribute, costs to add a new attribute authority. The DNF-MABS saves the cost in TR_MABS using a disjunctive normal form to express a policy.

According to the strong tendency that most Internet clients are implemented as a web based application, the same situation goes with the user interface for recent network-based information systems. In the security aspects, the web-based interface is considered much vulnerable in terms of the attack space and surface. In particular, the Cross Site Scripting attack was reported as the most vulnerable one against web applications, which is more than a half of all the reported practical attacks. The last paper [6] "Detection of Cross Site Scripting Attack in Wireless Networks using n-Gram and SVM" from Jun-Ho Choi, Chang Choi, Byeong-kyu Ko and Pan-Koo Kim introduces a framework that uses a Support Vector Machine based classification method to detect malicious script code, especially of the SQL injection attack. In the proposed framework, collections of scripts are preprocessed through the n-Gram indexing to generate the training data, and then are applied to the SVM with the code dictionary to learn the patterns and match the results. The paper proves that the proposed framework gains more than 5\% in performance against the existing filtering algorithms through a comparison of the test results.

Finally, we would like to express our special thanks to all authors and reviewers for their countless contribution and dedication to this special issue. We are also grateful to Professor David Taniar, the Editor-in-Chief of Mobile Information System, for supporting us to have made this special issue a reality.

\section{References}

[1] A.J. Jara, M.A. Zamora and A.F.G. Skarmeta, Glowbal IP: an adaptive and transparent IPv6 integration in the Internet of Things, Mobile Information System (MIS), 2012.

[2] F. Palmieri and A. Castiglione, Condensation-Based Routing in Mobile Ad-Hoc Networks, Mobile Information System (MIS), 2012.

[3] X. Zhang, C. Jing, F. Tang, S. Fowler, H. Cui and X. Dong, Joint Redundant and Random Network Coding for Robust Video Transmission over Lossy Networks, Mobile Information System (MIS), 2012.

[4] Y. Shen, M. Wang, X. Tang, Y. Luo and M. Guo, Context-aware HCI Service Selection, Mobile Information System (MIS), 2012.

[5] D. Cao, B. Zhao, X. Wang and J. Su, Flexible Multi-Authority Attribute-based Signature Schemes for Expressive Policy, Mobile Information System (MIS), 2012.

[6] J.-H. Choi, C. Choi, B.-K. Ko and P.-K. Kim, Detection of Cross Site Scripting Attack in Wireless Networks using n-Gram and SVM, Mobile Information System (MIS), 2012. 

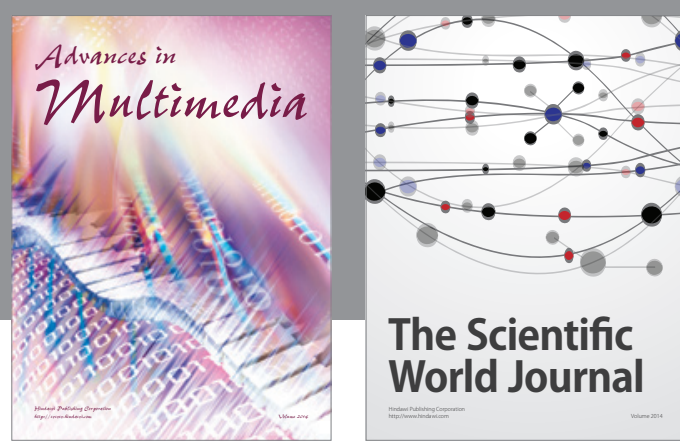

The Scientific World Journal
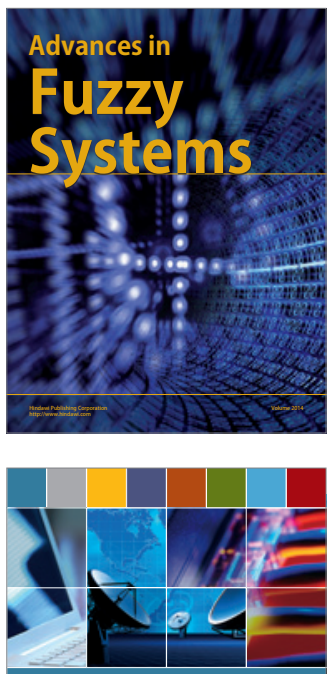

Computer Networks and Communications
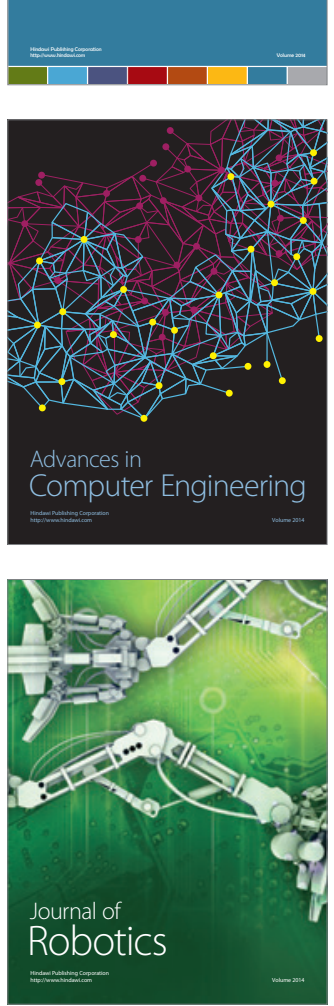
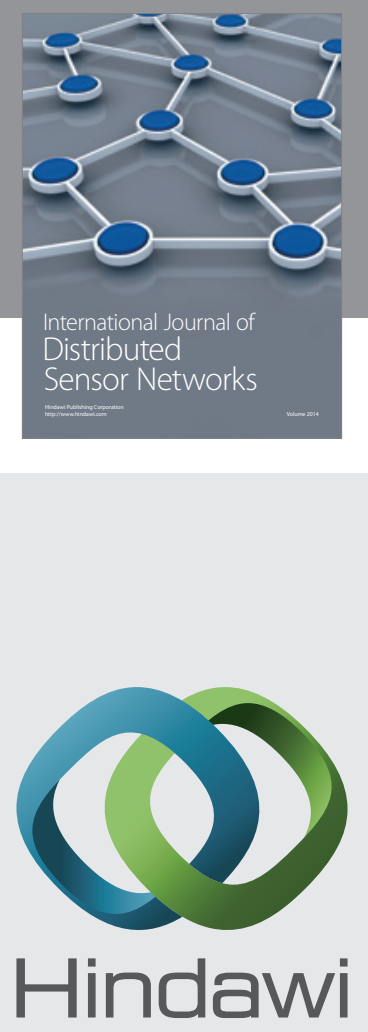

Submit your manuscripts at

http://www.hindawi.com
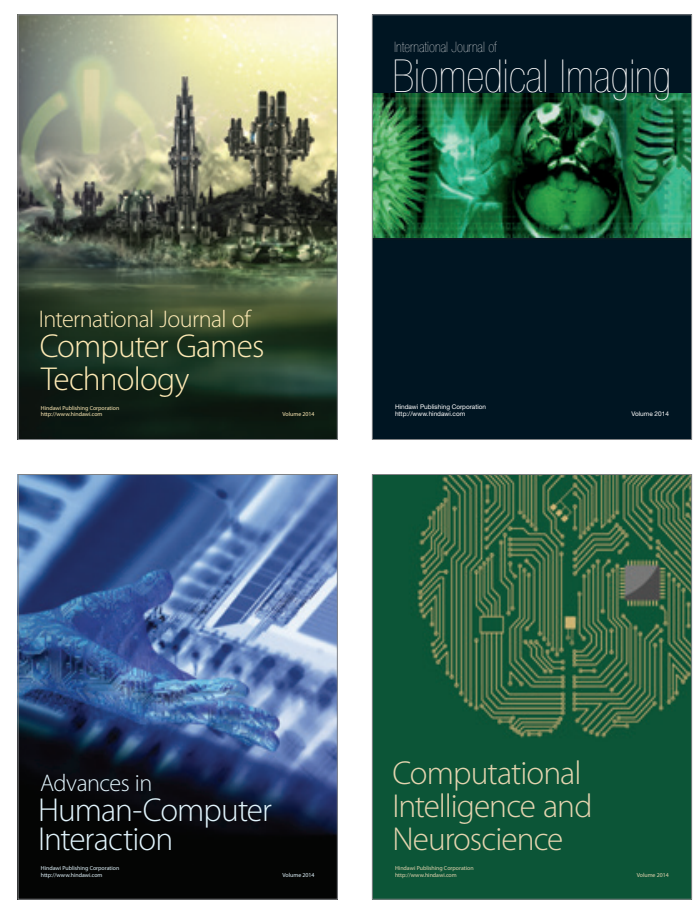
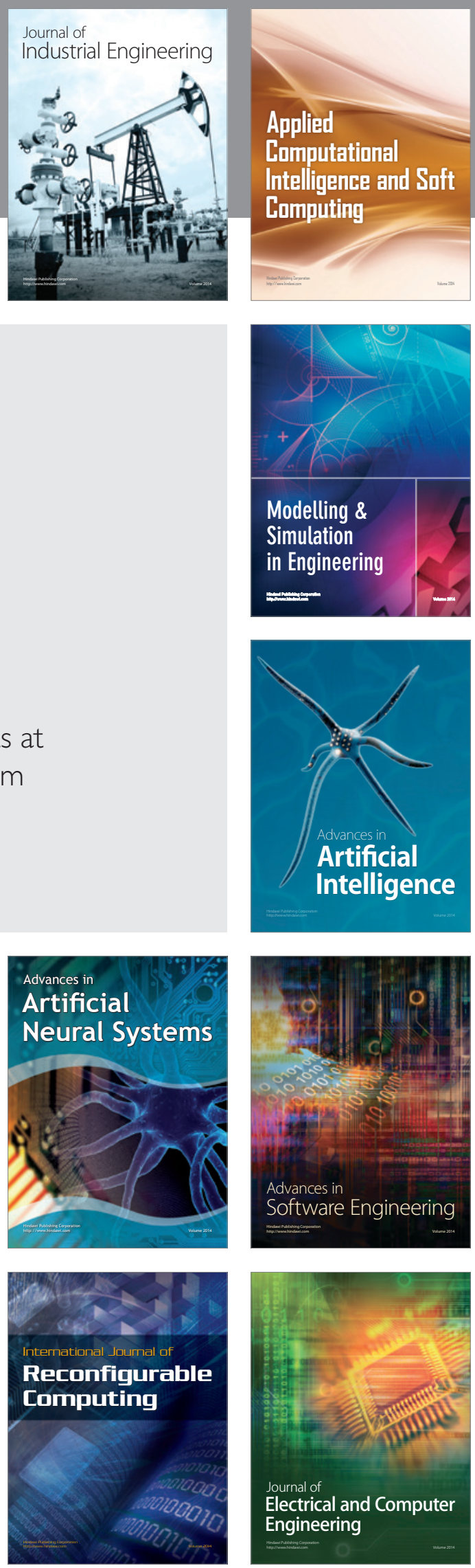